\title{
Commentary: Craving in Opioid Use Disorder: From Neurobiology to Clinical Practice
}

\author{
Rui Zheng ${ }^{1+}$, Liu Hao ${ }^{2 \dagger}$, Yuanhui Li ${ }^{1}$, Tianjiao Zhang ${ }^{1}$, Dexiang Bai ${ }^{1}$, Liqun Zhang ${ }^{1}$, Dai Li ${ }^{1}$ \\ and Wei $\mathrm{Hao}^{3 *}$ \\ ${ }^{1}$ Adai Technology (Beijing) Ltd., Co, Beijing, China, ${ }^{2}$ Department of Clinical Laboratory, Second Xiangya Hospital, Central \\ South University, Changsha, China, ${ }^{3}$ Mental Health Institute of the Second Xiangya Hospital, National Clinical Research \\ Center on Mental Disorders Central South University, Changsha, China
}

Keywords: craving, objectively assessing, virtual reality, machine learning, biomarker

\section{A Commentary on}

Craving in Opioid Use Disorder: From Neurobiology to Clinical Practice by Kakko, J., Alho, H., Baldacchino, A., Molina, R., Nava, F. A., and Shaya, G. (2019). Front. Psychiatry. 10:592. doi: 10.3389/fpsyt.2019.00592

\section{OPEN ACCESS}

Edited by:

Jianhua Chen,

Shanghai Jiao Tong University, China

Reviewed by:

Ti-Fei Yuan,

Shanghai Jiao Tong University, China

${ }^{*}$ Correspondence:

Wei Hao

weihao57@csu.edu.cn

weihao57@163.com

tThese authors have contributed equally to this work and share first

authorship

Specialty section:

This article was submitted to Addictive Disorders,

a section of the journal

Frontiers in Psychiatry

Received: 10 October 2020

Accepted: 05 July 2021

Published: 09 August 2021

Citation:

Zheng R, Hao L, Li Y, Zhang T, Bai D,

Zhang L, Li D and Hao W (2021)

Commentary: Craving in Opioid Use Disorder: From Neurobiology to

Clinical Practice.

Front. Psychiatry 12:615921. doi: 10.3389/fpsyt.2021.615921

\section{INTRODUCTION}

We read with great interest the review article by Kakko et al. (1), which focused on the opioid use disorder-related craving. A recent review also discussed underlying neurobiological changes in opioid use disorder (OUD) that likely contribute to drug craving (2). However, how to assess the craving in clinical practice is challenging. Here, we would like to present the recent developments and briefly introduce our work on objectively assessing craving.

\section{Current Practice of Evaluating Drug Addiction Cravings and the Limitations}

Craving is the central concept of almost all major drug dependence models (3-7). In the International Classification of Diseases [ICD-11 (8)], craving is listed as one of the six characteristics of psychoactive substance dependence. In DSM-5 $(9,10)$, craving has once again become the recommended standard for diagnosing substance use disorder (SUD).

Current craving assessments are often accomplished through self-reports and observations of cognitive performance (11).

Despite the development of several psychometrically validated multi-item craving inventory tools $(12,13)$, self-reported cravings are still most commonly evaluated using a single item craving inventory (11). Since the intensity, latency, frequency, and significance of craving episodes may vary (14), self-reported assessments may have difficulty in reflecting true differences of cravings among patients (15). In daily clinical experiences, denial is one of the typical responses when we ask patients with alcohol and drug problems for their addictive behaviors, and in certain situations, for instance, in order to gain acceptance from family members, or gain enough trust to seek drugs privately, patients may conceal the true level of cravings.

Cognitive performance tasks are used to implicitly assess cravings $(6,16)$, based on the assumption that craving leads to the redistribution of cognitive processing resources and shifts that to drug-related cues (6). Therefore, facing the drug-related cues at a certain intensity, people with different intensities of drug addiction cravings would show different cognitive performances. Through recording cognitive performance parameters (e.g., the reaction time), it could reflect the situations of redistribution of cognitive processing resources, and therefore indirectly reflect the 
related craving. This indirect assessment method may avoid subjective bias (15) and may help to understand how craving changes perceptions and decisions. However, it is very challenging to implement in clinical practice $(11,12)$.

Kakko et al. (1) also mentioned using ecological momentary assessments (EMA) to assess cravings $(17,18)$. EMA uses mobile technology to record a real-time (including daily, random, or event-triggered) cravings (17-20). However, EMA only improves the understanding of craving-related time fluctuations $(17,18)$, but fail to improve craving assessment itself.

\section{Current Approach of the Assessment of Induced Cravings and the Limitations}

In addition to Background cravings (7), cravings can also be induced through specific drug-related prompts or stressful life events (7). Most craving-inducing studies use the cue-reactivity paradigm (7), in which drug users respond differently to drugrelated cues, in contrast with neutral cues, resulting in changes in brain activity related to the degree of craving (7).

The way to induce cravings at present depends mainly on the contents of pictures or videos. Although related to drug-seeking behavior and successfully induced cravings, they may not trigger reactions representing the real social or environmental cues (21), of which studies have shown that the treatment requires intervention (21).

\section{New Approach to Evaluating Drug Addiction Cravings With New Technology}

Our research team has designed the VR paradigm $(22,23)$ to improve the way to induce cravings, which can simulate the real world with the implicit cue, rebuild specific environments and characters, and allow certain social immersion and interaction, as well as the balanced drug-related cue and neutral stimuli. By technically arranging the sequence of stimuli and social interactions in the scenes, an effective evaluation experimental paradigm is formed.

Our results showed that the effects of different cues are related to the types of drug dependence, and VR stimulation could cause a higher level of cravings than the traditional picture and video stimulations, consistent with previous findings $(14,24)$. Through different types of induced stimuli, specific incentives for craving can provide a valuable therapeutic reference for clinical consultation and treatment.

During VR stimulation, we recorded several physiological parameters, including electroencephalogram (EEG), galvanic skin response, and heart rate $(22,23)$. We employed machine learning methods to analyze these physiological data to distinguish drug-dependent individuals from normal controls $(22,23)$.

We used machine learning algorithms, including random forest, AdaBoost, logistic regression, and ensemble model to build classification models $(22,23)$. The most striking findings were that the highest classification accuracy of $86 \%$ was obtained by the AdaBoost algorithm, with $84 \%$ precision, $83 \%$ recall rate, and $83 \%$ f1 score (23). Our results show that compared with traditional statistical methods, machine learning has advantages in processing massive and complex physiological data, which helps identify features that can distinguish different populations.

\section{DISCUSSION}

The level of craving is unstable and affected by multiple factors and changes along with the internal and external environments over time, while the patient's dependent state is relatively stable. Thus, it is possible to find stable biomarkers reflecting a patient's status related to dependence rather than reflecting the real-time craving level. This is urgently needed for clinical practice, not only for objective diagnosis and severity of dependence but also to be used as an early sign for relapse.

Because of the complexity of mental illnesses such as addiction, the traditional research methods based on reductionism were limited and overwhelmed (25). The emergence of new technologies, such as machine learning, has made holistic research possible in this field. The previous reductionist studies are hypothesis-driven; that is, they explore the regularity based on a relatively clear hypothesis. Such studies often focus on specific mechanisms, and the results are highly explanatory, while machine learning studies are driven by holistic data. Through in-depth mining of overall data, machine learning studies could provide more detailed results and new directions, although the interpretability is sometimes poor.

The advantage of the holism method should be considered with the help of multidisciplinary forces and high-level technology, such as biophysics, non-linear computing, and machine learning. Using holism methods to find the regularity first and then exploring the specific mechanism may be a promising solution. We developed a model that could accurately distinguish drug-dependent individuals and normal controls by using machine learning algorithms, obtained potential key biomarkers of the dependent state, and achieved some promising results (26).

At the same time, we explored the correlation between the probability results of machine learning algorithms and some other clinical indicators, including not only the subjective craving inventory score but also drug use-related indicators, such as drug use duration, dosage, and frequency. Since the correlation could be intricate, we track and analyze most of the relevant indicators. Even though the research may not yield satisfying results in the short term, using machine learning to classify the population is meaningful in itself and provides a direction for further exploration of the biological nature of dependence and addiction.

\section{AUTHOR CONTRIBUTIONS}

WH initiated the project and designed the work. RZ and LH were approached to write the paper, structured it, and wrote sections Introduction and Current Practice of Evaluating Drug Addiction Cravings and the Limitations. YL and TZ wrote sections Current Approach of the Assessment of Induced cravings and the Limitations and New Approach to Evaluating 
Drug Addiction Cravings With New Technology. DB and LZ wrote section Discussion. RZ, LH, DL, and WH revised the manuscript. All authors contributed to the article and approved the submitted version.

\section{REFERENCES}

1. Kakko J, Alho H, Baldacchino A, Molina R, Nava FA, Shaya G. Craving in opioid use disorder: from neurobiology to clinical practice. Front Psychiatry. (2019) 10:592. doi: 10.3389/fpsyt.2019.00592

2. Lueptow LM, Shashkova EC, Miller MG, Evans CJ, Cahill CM. Insights into the neurobiology of craving in opioid use disorder. Curr Anesthesiol Rep. (2020) 10:378-387. doi: 10.1007/s40140-020-00420-7

3. Drummond DC. Theories of drug craving, ancient and modern. Addiction. (2001) 96:33-46. doi: 10.1046/j.1360-0443.2001.961333.x

4. Tiffany ST, Warthen MW, Goedeker KC. The functional significance of craving in nicotine dependence. Nebr Symp Motiv. (2009) 55:17197. doi: 10.1007/978-0-387-78748-0_10

5. Baker TB, Piper ME, McCarthy DE, Majeskie MR, Fiore MC. Addiction motivation reformulated: an affective processing model of negative reinforcement. Psychol Rev. (2004) 111:3351. doi: 10.1037/0033-295X.111.1.33

6. Tiffany ST. A cognitive model of drug urges and drug-use behavior: role of automatic and nonautomatic processes. Psychol Rev. (1990) 97:14768. doi: 10.1037/0033-295X.97.2.147

7. Tiffany ST, Wray JM. The clinical significance of drug craving. Ann N Y Acad Sci. (2012) 1248:1-17. doi: 10.1111/j.1749-6632.2011.06298.x

8. ICD-11: International Classification of Diseases 11th Revision. Geneva: World Health Organization (2018). Available online at: https://icd.who.int/browse11

9. American Psychiatric Association Task Force On DSM-V. Diagnostic and Statistical Manual of Mental Disorders: DSM-V. 5th ed. (2013). doi: 10.1176/appi.books.9780890425596

10. Hasin DS, O’Brien CP, Auriacombe M, Borges G, Bucholz K, Budney A, et al. DSM-5 criteria for substance use disorders: recommendations and rationale. Am J Psychiatry. (2013) 170:834-51. doi: 10.1176/appi.ajp.2013.12060782

11. Rosenberg H. Clinical and laboratory assessment of the subjective experience of drug craving. Clin Psychol Rev. (2009) 29:51934. doi: 10.1016/j.cpr.2009.06.002

12. Sayette MA, Shiffman S, Tiffany ST, Niaura RS, Martin CS, Shadel WG. The measurement of drug craving. Addiction. (2000) 95(Suppl. 2):S189210. doi: 10.1046/j.1360-0443.95.8s2.8.x

13. McHugh RK, Fitzmaurice GM, Carroll KM, Griffin ML, Hill KP, Wasan AD, et al. Assessing craving and its relationship to subsequent prescription opioid use among treatment-seeking prescription opioid dependent patients. Drug Alcohol Depend. (2014) 145:121-6. doi: 10.1016/j.drugalcdep.2014.10.002

14. Hone-Blanchet A, Wensing T, Fecteau S. The use of virtual reality in craving assessment and cue-exposure therapy in substance use disorders. Front Hum Neurosci. (2014) 8:844. doi: 10.3389/fnhum.2014.00844

15. Sayette MA. The role of craving in substance use disorders: theoretical and methodological issues. Annu Rev Clin Psychol. (2016) 12:40733. doi: 10.1146/annurev-clinpsy-021815-093351

16. Field M, Munafo MR, Franken IH. A meta-analytic investigation of the relationship between attentional bias and subjective craving in substance abuse. Psychol Bull. (2009) 135:589-607. doi: 10.1037/a00 15843

17. Serre F, Fatseas M, Swendsen J, Auriacombe M. Ecological momentary assessment in the investigation of craving and substance use in daily life: a systematic review. Drug Alcohol Depend. (2015) 148:1-20. doi: 10.1016/j.drugalcdep.2014.12.024

\section{FUNDING}

This work was supported by the National Key R\&D Program of China (No. 2017YFC1310400).

18. Huhn AS, Harris J, Cleveland HH, Lydon DM, Stankoski D, Cleveland MJ, et al. Ecological momentary assessment of affect and craving in patients in treatment for prescription opioid dependence. Brain Res Bull. (2016) 123:94-101. doi: 10.1016/j.brainresbull.2016.01.012

19. Moskowitz DS, Young SN. Ecological momentary assessment: what it is and why it is a method of the future in clinical psychopharmacology. J Psychiatry Neurosci. (2006) 31:13-20.

20. Preston KL, Jobes ML, Phillips KA, Epstein DH. Real-time assessment of alcohol drinking and drug use in opioid-dependent polydrug users. Behav Pharmacol. (2016) 27:579-84. doi: 10.1097/FBP.0000000000000250

21. Perry JL, Joseph JE, Jiang Y, Zimmerman RS, Kelly TH, Darna M, et al. Prefrontal cortex and drug abuse vulnerability: translation to prevention and treatment interventions. Brain Res Rev. (2011) 65:12449. doi: 10.1016/j.brainresrev.2010.09.001

22. Ding X, Li Y, Gong L, Li D, Peng Z, Bi Y, et al. A machine learning approach to detect methamphetamine craving induced in a virtual reality environment. In: AAAP 29th Annual Meeting and Scientific Symposium. Bonita Springs, FL (2018).

23. Ding X, Li Y, Qi Y, Bi Y, Tang H, Li D, et al. A machine learning approach using EEG data to detect methamphetamine craving induced in a virtual reality environment. In: Society for Neuroscience 2018-Behavioral Studies of Amphetamines. San Diego, CA (2018).

24. McHugh RK, Fulciniti F, Mashhoon Y, Weiss RD. Cue-induced craving to paraphernalia and drug images in opioid dependence. Am J Addict. (2016) 25:105-9. doi: 10.1111/ajad.12344

25. Choudhury S, Slaby J. Critical Neuroscience: A Handbook of the Social and Cultural Contexts of Neuroscience. Re-Socializing Psychiatry. (2011). p. 30530. doi: 10.1002/9781444343359.ch15

26. Ding $\mathrm{X}, \mathrm{Li}$ Y, Li D, Li CL, Liu, X*. Using machine learning approach to distinguish patients with methamphetamine dependence from healthy subjects in a virtual reality environment. Brain Behav. (2020) 10:e01814. doi: $10.1002 /$ brb3.1814

Conflict of Interest: RZ, YL, TZ, DB, LZ, and DL were employed by the company Adai Technology (Beijing) Ltd., Co.

The remaining author declares that the research was conducted in the absence of any commercial or financial relationships that could be construed as a potential conflict of interest.

Publisher's Note: All claims expressed in this article are solely those of the authors and do not necessarily represent those of their affiliated organizations, or those of the publisher, the editors and the reviewers. Any product that may be evaluated in this article, or claim that may be made by its manufacturer, is not guaranteed or endorsed by the publisher.

Copyright (c) 2021 Zheng, Hao, Li, Zhang, Bai, Zhang, Li and Hao. This is an open-access article distributed under the terms of the Creative Commons Attribution License (CC BY). The use, distribution or reproduction in other forums is permitted, provided the original author(s) and the copyright owner(s) are credited and that the original publication in this journal is cited, in accordance with accepted academic practice. No use, distribution or reproduction is permitted which does not comply with these terms. 